\title{
Modeling of Electric Vehicle Chargers
}

\author{
Marco S.W. Chan, K.T. Chau and C.C. Chan \\ Department of Electrical \& Electronic Engineering \\ The University of Hong Kong \\ Pokfulam, Hong Kong, China \\ ktchau@eee.hku.hk
}

\begin{abstract}
The chargers of electric vehicles (EV) are highly nonlinear devices. They are characterized by high harmonic current distortion and poor power factor. Different models of chargers and converters had been studied and a number of papers were published. In order to study the impacts of a large number of electric vehicles to the power system, an appropriate charger model for a population of EVs should be developed. To illustrate the process in using Monte Carlo method to describe the interaction of $\mathrm{EV}$ chargers, the mean value of the total harmonic distortion (THD) is estimated as it is one of the most important parameters should be studied. Different charging algorithms will have different performance and harmonic distortion. Since current-voltage control charging method is widely adopted for various types of batteries, a model based on this algorithm is presented.
\end{abstract}

\section{INTRODUCTION}

With ever increasing concerns on energy efficiency, energy diversification and environmental protection, the development of electric vehicle (EV) technology has taken on an accelerated pace. The dream of having commercially viable EVs is becoming a reality [1]-[5].

The first practical EV was built in Britain by Robert Davidson in 1873, whereas the first gasoline-powered vehicle did not appear until 1885 , nearly 12 years later. With the drastic improvement in the internal combustion engine, gasoline-powered vehicles showed much better performance and the use of EVs were almost absent from the 1930s to the 1950s. The rekindling of interests in EVs started at the outbreak of energy crisis and oil shortage in the 1970s. Over a single year between 1973 and 1974, the price of a barrel of oil increased fourfold. The actual revival of EVs should be due to the ever increasing concerns on energy conservation and environmental protection throughout the world:

- EVs offer high energy efficiency. In general, the overall energy conversion efficiencies from crude oil to vehicle motion for EVs and gasoline-powered vehicles are $12.5 \%$ and $9.3 \%$, respectively. Moreover, EVs can perform efficient braking by converting the kinetic energy back to electricity.

- EVs allow energy diversification. Electricity can be generated not only from thermal power using oil and coal, but also from hydro power, wind power, geothermal power, wave/tidal power, solar power and nuclear power.

- EVs enable load equalization of power system. By recharging EVs at night, those non-stockable energy at non-peak hours and hence the power generation facilities can be effectively utilized, contributing to energy saving and stabilization of power cost.

- EVs show zero local exhaust emissions. Even globally, the emissions due to the generation of electricity for EVs are only $2 \%$ in carbon monoxide, $76 \%$ in carbon dioxide, $56 \%$ in nitrogen oxides and $9 \%$ in hydrocarbons exhausted by gasoline-powered vehicles. Increasingly, the emissions generated by power plants can be further minimized by dedicated filtering, and even recycled such as the use of carbon dioxide recycling for electricity generation.

- EVs operate quietly and almost vibration-free, whereas gasoline-powered vehicles are inherently noisy and with sensible vibration. Thus, EVs are welcomed by drivers and appreciated by local residents.

Consequently, some governments have enforced stricter emissions regulations encouraging the promotion of EVs. In October 1990, the California Air Resources Board established rules that $2 \%$ of all vehicles sold in the state between 1998 and 2002 be emission-free, and $10 \%$ of vehicles put on the market must have zero emissions by 2003. In 1996, the Board decided to scrap the $2 \%$ mandate since there is no way for the automobile industry to meet that timetable, and to retain the $10 \%$ mandate by 2003 . Response to this mandate is sure to be a major issue among automobile manufacturers around the world.

\section{EV CHARGING}

The challenge of transforming EVs from concept to reality is to make it safe, convenient and easy for consumers to charge batteries. In order to improve convenience and increase charging efficiency, a number of charging schemes have been proposed: home charge, regenerative charge, solar charge, park-and-charge (PAC), and move-and-charge (MAC).

As an EV is usually parked at home or a storage site at night, the battery charger in the vehicle can be connected into the domestic single-phase AC plug for slow night-time charging. Depending on the battery capacity and depth of discharge, the charging time takes about 6-8 h, and the charging current is usually limited to $15 \mathrm{~A}$. As the electricity demand at night is relatively low, this home charging scheme can facilitate the load level control of power utilities.

During deceleration or down-hill, the EV propulsion motor is operated as a generator to charge batteries through regenerative braking, so-called regenerative charge. This can extend the driving range by up to $25 \%$. 
When an EV is parked at a charging station, a microprocessor-controlled three-phase off-board battery charger initiates the power and effects its transfer to the vehicle. When an amount of parking time selected is insufficient to supply an amount of power selected using a normal charging scheme, the intelligent off-board charger allows for quick charge by adjusting continually the charging rate to match the ability of batteries to accept charge. During quick charge, the charging current is generally over $100 \mathrm{~A}$ so that the charging time is about 20 min to attain $80 \%$ state-of-charge. It should be noted that from the power utility point of view, quick charge may not be desirable because it causes high peak power demand. An incentive-based electricity billing system may be employed to encourage people to charge batteries during off-peak periods, while quick charge is encouraged only for emergency purposes at dedicated charging stations.

The most ideal situation for charging EV batteries is to perform charge while the vehicle is cruising on the road - socalled MAC. Thus, the driver does not need to find a charging station, park the vehicle, and spend relatively long time to charge up batteries. This MAC system is embedded on the surface of a section of highway, the charging zone, and does not need any additional space. Both contact and inductive types of MAC can be implemented. For the contact type MAC system, an on-board contact arch is mounted on the bottom of the EV body. By physically contacting the charging elements which have been embedded on the road surface, the arch picks up instantaneous high current. Since the EV is cruising through the charging zone, the charging process is so-called pulse charge. For the inductive type MAC system, the on-board contact arch is replaced by inductive coils, and the charging elements are replaced by high current coils which produce strong magnetic field.

By estimating the present number of gas stations for gasoline-powered vehicles, the demand on battery charging stations for EVs must be enormous. Thus, the development of battery chargers plays a very important role for preparing the charging infrastructure of EVs.

With the ever increasing popularity on using rechargeable batteries, the development of battery chargers are accelerated. Apart from concentrating on the development of the converter topologies for battery chargers such as soft-switching ac-dc converters, some developments on the harmonic modeling and analysis of battery chargers have been conducted. Unlike ordinary inductive or capacitive load, the battery chargers are non-linear devices due to the presence of power semiconductors such as diodes or SCRs. In additional to poor power factor, they do impose harmonic currents to the power system. The total harmonic current distortion is dependent on the charging algorithm, which ranges from $5 \%$ to $100 \%$ [6]. Without appropriate compensation, such harmonic current distortion causes serious problems on the accuracy of revenue billing meter, voltage distortion and even system instability [7].

When a large of population of EVs is adopted, the corresponding harmonics impact to the power system is expected to be serious. However, the available approaches to the analysis of power quality are generally based on a standalone converter, and cannot be directly used to describe the net effect of a population of EVs. For example, there should be a number of EVs being charged at the same moment with different state-of-charge (SOC) at a particular charging station. The net current demand, power factor and harmonics of each $\mathrm{EV}$ are different from each other.

There are a number of criteria like IEEE Standard 519 has been issued to restrict the generation of harmonics [8]. The net effect of a population of EVs is not merely the numerical sum of the total harmonic distortion THD, which involves both the magnitudes and phase angles of individual harmonic components. Moreover, the parameters change in according to the SOC of individual EVs - leading to be a stochastic problem.

In this paper, a charger model is developed in such a way that it can estimate the corresponding THD in the presence of a population of EVs. As the current-voltage control charging algorithm is suitable for a variety of batteries such as lead-acid battery and nickel-cadmium battery, this algorithm is adopted for simulation. The corresponding stochastic problem is handled by using Monte Carlo method.

\section{MONTE CARLO METHOD}

Monte Carlo method is a statistical simulation method that utilizes sequences of random numbers to perform simulation [9], [10]. The method is different from the conventional numerical discretization methods. There is no need to write down the differential equations that describe the behavior of the system. The only requirement is to describe it by probability density functions $(p d f)$. Once these $p d f$ are known, the Monte Carlo process can be carried out by sampling randomly from these $p d f$. Then, a large number of simulations are performed, and the average value of the observations is computed to get the desired result.

To study the impacts of a population of EVs," a particular state, namely the battery voltage of an $\mathrm{EV}$, is represented by a random variable $X$ and the $p d f$ of $X$ as represented by $f(x)$. The value of current demand can then be expressed as a function of the random variable $X$, and is represented as $g(X)$. For a number of EVs, the mean value of the function is expressed as:

$\mu[g(X)]=\sum_{i=1}^{k} f\left(x_{i}\right) g\left(x_{i}\right)$

and the variance is given by:

$\sigma^{2}[g(X)]=\sum_{i=1}^{k} f\left(x_{i}\right) g^{2}\left(x_{i}\right)-\mu^{2}[g(X)]$

Obviously, $g(X)$ is also a random variable. According to the rule of three sigmas in [8], the following probability:

$$
P\left\{\left|\frac{1}{N} \sum_{i=1}^{N} g\left(x_{i}\right)-\mu[g(X)]\right|<\frac{3 b}{\sqrt{N}}\right\} \approx 0.997
$$

shows that the estimated standard error can be reduced by increasing the sample size $N$. However, the larger the sample size, the longer the simulation time is required. Anyway, this sample size can be bounded to a certain value. 


\section{OPERATION OF EV CHARGERS}

A $380 \mathrm{~V}$ single-phase EV charger is used for exemplification. It is employed to charge an EV which consists of a pack of lead-acid battery with 125 cells. The voltage level of each cell is $1.75 \mathrm{~V}$ in discharged state, and $2.4 \mathrm{~V}$ in fully charged state. Thus, the battery voltage ranges from $218 \mathrm{~V}$ to $300 \mathrm{~V}$ in accordance with the SOC. For the current-voltage control charging method, the charging current is kept constant at a value of about $0.2 \mathrm{C}$ to $0.3 \mathrm{C}$ of the battery capacity. When the battery voltage rises to the gassing value, the output voltage of the charger is kept constant, and the current from this point falls rapidly. The block diagram of this charger is shown in Fig. 1 which is composed of a rectifier, a converter and the battery pack for an EV.

Since the converter of the charger is of constant current output, it can be represented by a current source. Usually the switching frequency of this converter is above $20 \mathrm{kHz}$ so as to effectively eliminate those high-frequency components by a line filter. Therefore, the influence of high switching frequency can be ignored during the study of the harmonic current distortion. With a reasonable size of inductor, the input current waveform can be considered to be rectangular as shown in Fig. 2. Applying Fourier analysis, the input current can then be expressed as:

$$
\begin{aligned}
I= & \sum\left\{\frac { 1 } { n \pi } \left[\sin \left(n \cdot \beta_{2}\right)-\sin \left(n \cdot \beta_{1}\right)\right.\right. \\
& \left.\left.-\sin \left(n \cdot \beta_{4}\right)+\sin \left(n \cdot \beta_{3}\right)\right] \cdot \cos (n \omega \cdot t)\right\} \\
& +\sum\left\{\frac { 1 } { n \pi } \left[-\cos \left(n \cdot \beta_{2}\right)+\cos \left(n \cdot \beta_{1}\right)\right.\right. \\
& \left.\left.+\cos \left(n \cdot \beta_{4}\right)-\cos \left(n \cdot \beta_{3}\right)\right] \cdot \sin (n \omega \cdot t)\right\}
\end{aligned}
$$

where $\beta_{1}, \beta_{2}, \beta_{3}$ and $\beta_{4}$ are the conduction angles. The equation can be rewritten as:

$I=\sum c_{n} \cos \left(n \omega \cdot t+\varphi_{n}\right)$

where $c_{n}=\sqrt{a_{n}^{2}+b_{n}^{2}}$ and $\varphi_{n}=\tan ^{-1}\left(\frac{b_{n}}{a_{n}}\right)$, which are the magnitudes and phase angles of the harmonic components, respectively.

At a predetermined battery voltage of $300 \mathrm{~V}(2.4 \mathrm{~V}$ per cell for the lead-acid battery), the charging process is ceased in order to prevent over-charge damage to the battery. As the voltage drops across the converter and the rectifier are neglected and the current is assumed to flow when the ac voltage is greater than the battery voltage, the conduction angles $\beta_{1}, \beta_{2}, \beta_{3}$ and $\beta_{4}$ can be determined by the battery voltage $V_{\text {bat }}$ which is a function of SOC. Thus, the coefficients $a_{\mathrm{n}}, b_{\mathrm{n}}$ and $c_{\mathrm{n}}$ can be found out. Obviously, THD is a function of SOC or the battery voltage, and is represented by $g\left(V_{\text {bat }}\right)$.

Using (1) and (2), the THD of harmonic components (up to the 15-th harmonics) and their corresponding phase angles can be found. As shown in Fig. 3, the THD is a monotonic increasing function of the battery voltage, which agrees with our expectation because the input current waveform is distorted with the shortening of the conduction times. The maximum and minimum values of the THD are found to be $30.6 \%$ to $25.8 \%$, respectively. As the phase angles of individual harmonic components swing irregularly between $+\infty$ to $-\infty$, cancellation of harmonics may occur. To make the figures more readable, the vertical scale of phase angles is limited to $\pm 90^{\circ}$.

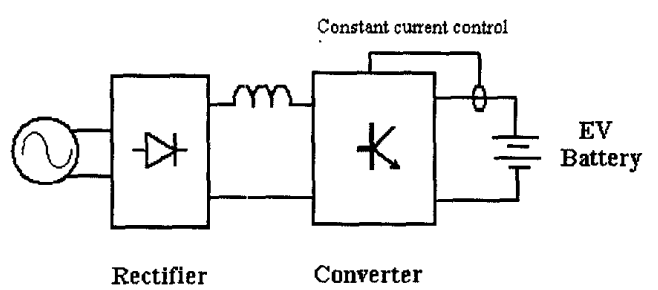

Fig. 1. Block diagram of charger.

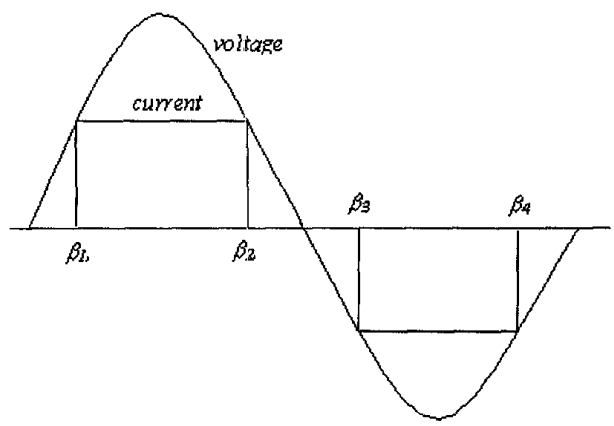

Fig. 2. Input voltage and current waveforms
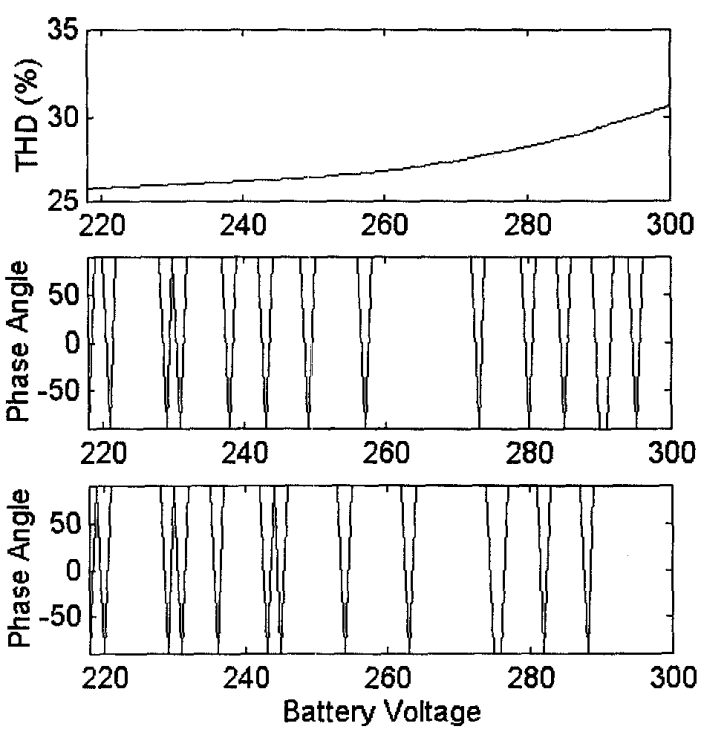

Fig. 3. Harmonic characteristics against battery voltage: (top) THD; (middle) phase angle of the 3-rd harmonic current; (bottom) phase angle of the 5-th harmonic current. 


\section{EFFECT OF A POPULATION OF EVS}

Once the expression for the model of an EV charger has been derived, the net effect of a population of EVs can be studied. As mentioned before, an EV charger is not only a source of harmonics but may also be a compensation device for another EV. As the phase angles of different harmonic components generated from an $\mathrm{EV}$ vary according to the individual SOC. It is expected that the THD would come to the minimum value if there were infinite number of EVs being charged at the same time. In the real world, only finite outlets can be provided at a particular station for EV charging, and only limited number of EVs can be found at a station. For those charging stations, which are far away from each other and separated by lengthy transmission lines with considerable impedance $Z$, should be considered independently.

As shown in Fig. 4, the net harmonic current injected to the power system from a charging station with $k \mathrm{EVs}$ is investigated. After the net THD with different number of EVs being charged at the station has been evaluated, an optimal value for the number of EVs that a charging station should accommodate can then be determined.

Now the number of EVs being charged per station is represented by a finite number $k$, and $k$ random numbers are assigned to represent the SOC of these EVs. The battery voltages are represented by $\xi_{\mathrm{v} 1}, \xi_{\mathrm{v} 2}, \ldots, \xi_{\mathrm{vk}}$. At any instance, the EVs at a station will be charged to any different SOC and the battery voltage is assumed to be a linear function of SOC. Thus, the value of voltage spreads over the interval between $218 \mathrm{~V}$ and $300 \mathrm{~V}$ with uniform distribution and the corresponding probability density function can be represented by Fig. 5. The harmonics and the corresponding phase angles of each EV are defined by (4). To find out the net effect, all the corresponding coefficients are summed up and the net harmonics can be calculated by (5). The average value is simply obtained by dividing the total value with $k$. By the law of large number [9], the expected value of the harmonics can be estimated by repeating the procedures for $N$ times. Since the current $I$ is a function of conduction angles which are determined by the EV battery voltage, the THD can then be denoted by $g\left(\xi_{V}\right)$. Thus, the expected value of THD is given by:

$E[T H D(\%)]=\frac{1}{N} \sum_{j=1}^{N}\left\{\frac{1}{k} \sum_{i=1}^{k} f\left(\xi_{i, j}\right) g\left(\xi_{i, j}\right)\right\}$

In case $N$ is large enough, the central limit theorem asserts that the sum of $N$ independent random variables has an approximately normal distribution. In practical cases, $N$ $\geq 25$ can be considered as effectively large [10]. Therefore, the simulation is performed with different sample sizes, namely $N=25,100,500$ and 1000 for. After normalization, the corresponding THD is plotted in Fig. 6 for different numbers of EVs per charging station. From this figure, it can be easily found that the THD decreases as the number of EVs being charged per station increases, and it converges to an average of about $25.1 \%$.
Certainly, the THD can be minimized by encouraging infinite number of EVs to be charged at the same charging point. However, as shown in Fig. 6, a finite number of 10 EVs should be an optimal choice for this particular case because the corresponding THD is near the minimum while the handling of $10 \mathrm{EVs}$ in a limited charging area is realistic.

\section{A. Standard Error}

From Fig. 6, we observe that the curves seem to be more smooth as the sample size is increased from 25 to 1000 . The fluctuation of the curves with smaller sample size indicates that the standard error will be great as $N$ is less than 500 . However, it is not the case because the standard error cannot be further reduced greatly by just increasing $N$ as it is inversely proportional to the square root of the sample size. This can be revealed by the numerical values of the means and standard errors listed in Table I, in which it has been assumed that only $10 \mathrm{EVs}$ are being charged at the same charging station.

From the table, it can also be found that the reduction of the standard errors drop readily as sample size is greater than 100. It is interesting to note that the error rises a little bit when the sample size becomes 1000 . It indicates that the error cannot be always reduced by the increment of $N$. Theoretically, the standard error is inversely proportional to $\sqrt{ } N$. In reality, the reduction of the standard error is also dependent on other factors, such as the randomness of the pseudo-random number generated by the computer for simulation.

\section{B. Implication of Results}

It is shown that the THD is reduced to a certain value as more and more EVs are allowed to be charged at a particular point. Based on this result, it implies that the size of a charging station and the number of EVs which is expected to be accommodated can be determined. Moreover, the simulation results also imply that several nearby chargers, which are originally designed for charging only one or two EVs, should be grouped together and connected to the same bus-bar so that the harmonic distortion can be minimized. Similarly, several charging station with relatively low utilization should also be grouped together and fed by the same substation.

TABLE I

MEAN VALUE AND STANDARD ERROR OF THD WITH DIFFERENT SAMPLE SIZE

\begin{tabular}{ccc}
\hline Sample Size & Mean of THD(\%) & Standard Error \\
\hline 25 & 25.78 & 0.797 \\
100 & 25.39 & 0.605 \\
500 & 25.28 & 0.602 \\
1000 & 25.29 & 0.612 \\
\hline
\end{tabular}




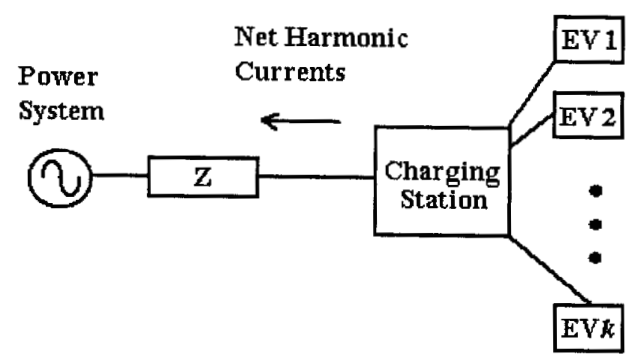

Fig. 4. Multiple EVs being charged at a charging station.

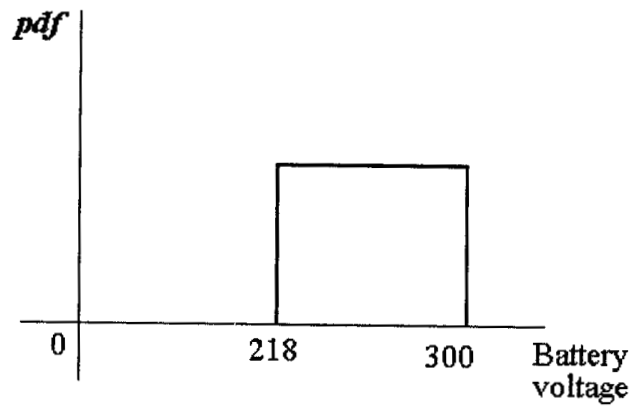

Fig. 5. Probability distribution function of battery voltage.

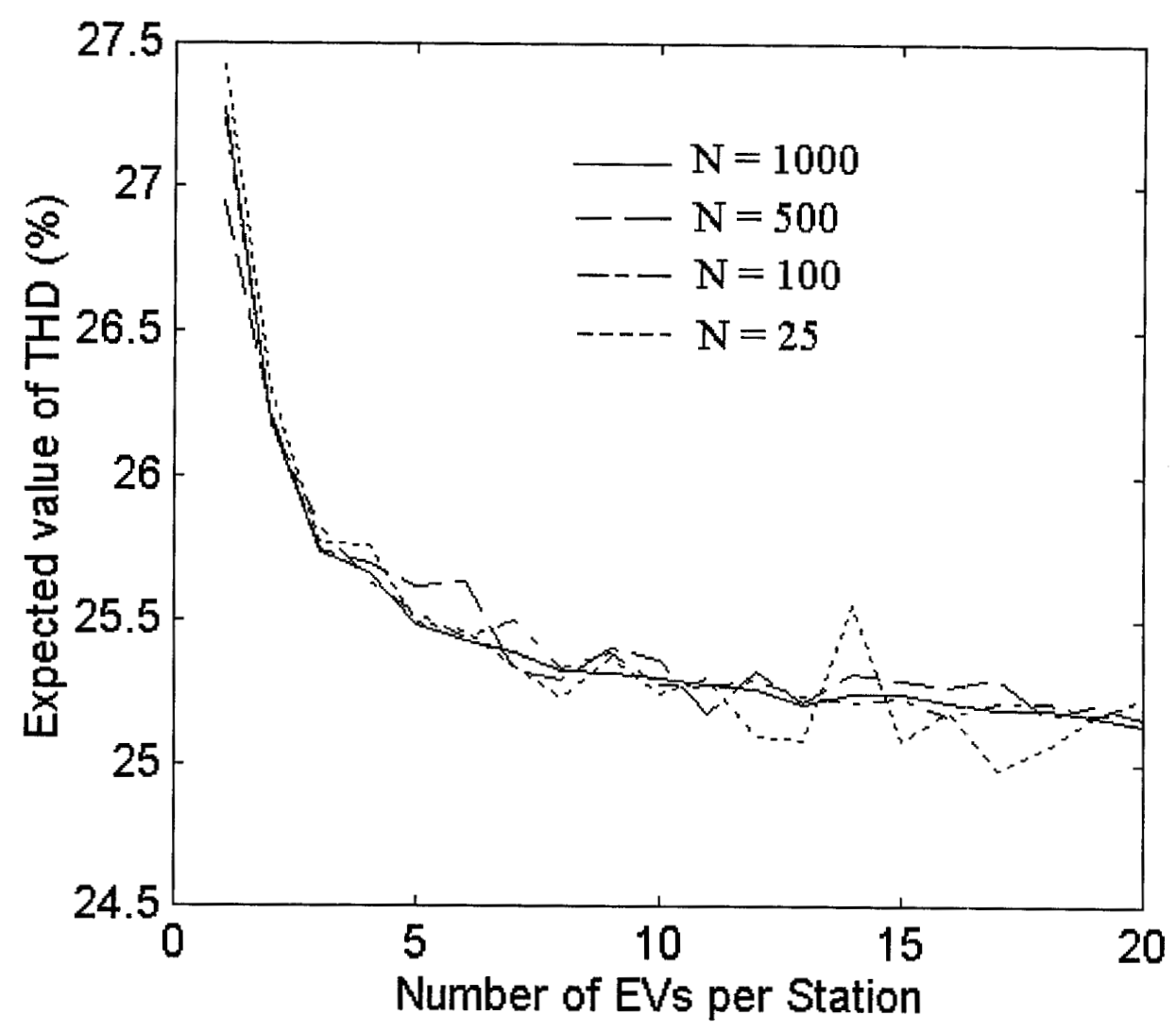

Fig. 6. Expected value of THD versus different number of EVs per station. 


\section{CONCLUSION}

The impacts of EVs to the power system are brought out, especially the inference of harmonic current is highlighted. This paper presents an idea that the total harmonic distortion can be minimized as more EVs are charged at the same charging station. A general approach which is based on the Monte Carlo method, is illustrated for the estimation of the system $\operatorname{THD}(\%)$. Although the result is based on an EV charger with a particular charging algorithm, the approach can readily be generalized for different chargers with various charging algorithms. Once the expression of different chargers can be derived, the expected value of the harmonics can also be determined. As a further extension, the impacts on average power factor and current demand can also be predicted by using the proposed approach.

\section{ACKNOWLEDGMENT}

This work was supported and funded in part by the Committee on Research and Conference Grants in the University of Hong Kong, and the Hong Kong Research Grants Council.

\section{REFERENCES}

[1] C.C. Chan and K.T. Chau, "An overview of power electronics in electric vehicles," IEEE Transactions on Industrial Electronics, Vol. 44, No. 1, February 1997, pp. 3-13.
[2] C.C. Chan and K.T. Chau, "Overview of electric vehicle technology and market potential in China," Proceedings of International Conference on Electric Vehicle Technology (EVT'95), Paris, November 1995, pp. 293-302.

[3] C.C. Chan and K.T. Chau, "An overview of electric vehicles - challenges and opportunities," Proceedings of IEEE Industrial Electronics Society Annual International Conference (IECON'96), Taipei, Taiwan, August 1996, pp. 1-6.

[4] C.C. Chan and K.T. Chau, "Review on modern drive technology for electric vehicles," IEEE Symposium on Industrial Drives and Traction (SIDT'96), Hong Kong, November 1996, pp. 3-14.

[5] C.C. Chan and K.T. Chau, "Power electronics and electric vehicles," Proceedings of International Conference on Power Electronics and Motion Control (PEMC'97), Hangzhou, China, November 1997, pp. 21-28.

[6] P.T. Staas, W.M. Grady, A. Aprapostathis and R.S. Thallam, "A statistical method for predicting the net harmonic current generated by a concentration of electric vehicle battery chargers," IEEE Transactions on Power Delivery, Vol. 12, No. 3, July 1997, pp. 1258-1266.

[7] R. Arseneau, G.T. Heydt, M.J. Kempker, "Application of IEEE Standard 519-1992 harmonic limits for revenue billing meters," IEEE Transactions on Power Delivery, Vol. 12, No. 1, January 1997, pp. 346-353.

[8] IEEE Standard 519 - 1992, Recommended Practices and Requirements for Harmonic Control in Electrical Systems, IEEE, New York, 1993.

[9] I.M. Sobol, A Primer for the Monte Carlo Method, CRC Press, 1994.

[10] J.M. Hammersley and D.C. Handscomb, Monte Carlo Methods, Chapman and Hall, 1979. 